\title{
Differentiation of histopathological growth patterns of colorectal liver metastases by MRI features
}

\author{
Wen-Hui Li ${ }^{1 \#}$, Shuai Wang ${ }^{2 \#}$, Yi Liu ${ }^{2}$, Xin-Fang Wang ${ }^{3}$, Yong-Feng Wang ${ }^{2}$, Rui-Mei Chai ${ }^{2} \wedge$ \\ ${ }^{1}$ Department of Gastrointestinal Onco-Pathology, First Hospital of China Medical University, Shenyang, China; ${ }^{2}$ Department of Radiology, First \\ Hospital of China Medical University, Shenyang, China; ${ }^{3}$ Department of Surgical Oncology, First Hospital of China Medical University, Shenyang, \\ China
}

Contributions: (I) Conception and design: RM Chai, S Wang; (II) Administrative support: Y Liu; (III) Provision of study materials or patients: WH Li, S Wang, XF Wang; (IV) Collection and assembly of data: S Wang, RM Chai; (V) Data analysis and interpretation: WH Li, RM Chai; (VI) Manuscript writing: All authors; (VII) Final approval of manuscript: All authors.

\#These authors contributed equally to this work.

Correspondence to: Rui-Mei Chai, PhD, MD. Department of Radiology, First Hospital of China Medical University, Nanjing North Street 155, Shenyang 110001, China. Email: chairuimei@sina.cn.

Background: It is necessary to develop an accurate non-invasive method to determine the histopathological growth pattern (HGP) of colorectal liver metastasis (CRLM) before surgery. The present study aimed to identify various HGPs of CRLM by magnetic resonance imaging (MRI) features.

Methods: This retrospective study included 53 chemo-naïve patients with CRLM between December 2013 and September 2019. The HGPs of CRLM were assessed according to the international consensus guidelines, and were classified as either replacement HGP (rHGP) or non-rHGP. The MRI features of CRLM were retrospectively reviewed in consensus by two radiologists. The differences of MRI features between rHGP and non-rHGP tumors were compared by using Chi-square test and Student's $t$-test. The Spearman or Pearson correlation analysis was performed to determine the correlation between different MRI features. A receiver operating characteristic (ROC) curve was plotted to evaluate the diagnostic ability.

Results: Of the 53 chemo-naïve patients (mean age, 60.11 \pm 9.85 years; age range, 38-86 years), 12 were diagnosed as rHGP, while 41 were diagnosed as non-rHGP. Rim enhancement were more common in rHGP than in non-rHGP $(\mathrm{P}<0.001)$. Besides, the diameter difference $(\Delta \mathrm{D})$ between the precontrast and postcontrast images of $r H G P$ was significantly larger than that of the non-rHGP $(\mathrm{P}=0.001)$. The rim width was correlated with $\Delta \mathrm{D}$, but not correlated with tumor size. The non-rHGP colorectal liver metastases were prone to be washed out in the delayed phases $(\mathrm{P}=0.043)$. The area under the curve (AUC) for the differentiation of rHGP and non-rHGP by using rim enhancement and $\Delta \mathrm{D}$ was 0.828 (95\% CI: 0.708-0.949). Conclusions: The MRI features of CRLM are characteristic and could help to differentiate rHGP and non-rHGP.

Keywords: Histopathological growth pattern (HGP); colorectal cancer; liver metastases; magnetic resonance imaging (MRI)

Submitted Feb 04, 2021. Accepted for publication May 06, 2021.

doi: $10.21037 /$ qims-21-143

View this article at: https://dx.doi.org/10.21037/qims-21-143

\footnotetext{
^ ORCID: 0000-0002-4553-2145.
} 


\section{Introduction}

The liver is the most common organ for colorectal cancer metastasis (1). Approximately $20 \%$ to $34 \%$ of patients with colorectal cancer present with synchronous liver metastasis at the time of diagnosis $(2,3)$. The presence of liver metastasis is an important prognostic factor and impacts the management of patients with colorectal cancer dramatically. Since 1990s, researchers have noticed the heterogeneity of liver metastasis growth pattern (4). However, the classification criteria and denominations of liver metastasis growth pattern tend to vary with time and studies (4-6). It was not until 2017 that the international consensus guidelines were proposed (7). According to the different interface between the metastatic cancer and the surrounding liver parenchyma, the colorectal liver metastasis (CRLM) mainly exhibits three different histopathological growth patterns (HGPs): desmoplastic, replacement, and pushing $(4,5,7)$. In the desmoplastic HGP (dHGP), the metastatic cancer cells are separated from the liver parenchyma by a rim of fibrous stroma, and there are no direct contact between cancer cells and hepatocytes. New blood vessels in the desmoplastic rim are formed by sprouting angiogenesis. In the replacement HGP (rHGP), metastatic cancer cells form cell plates that are in continuity with the liver cell plates, inducing co-option of sinusoidal blood vessels at the tumor-liver interface instead of sprouting angiogenesis. In the pushing HGP (pHGP), the liver cell plates that surround the metastasis are pushed away and compressed, without forming a surrounding desmoplastic rim, and the blood supply is also obtained by sprouting angiogenesis.

There is currently limited understanding of the biological mechanisms that underlie the different HGPs. Moreover, it is unclear why some tumors elicit a desmoplastic and angiogenic response while others grow in a co-opting manner and adopt the replacement growth pattern. However, the clinical significance of different HGPs has been proven. The rHGP is reported a significantly poor outcome for patients with this pattern, while the CRLM with dHGP is found a significantly better survival (6-10). The CRLM with rHGP responds poorly to the anti-angiogenic agent bevacizumab, which is recommended as one of the alternative chemotherapy drugs for the treatment of colorectal cancer (5). Additionally, the CRLM with rHGP suffers a higher intrahepatic recurrence rate after hepatectomy compared with its counterpart (6). Therefore, it is of clinically importance to determine the HGPs for the CRLM patients.

Although the classification of HGPs by light microscopy in standard hematoxylin-and-eosin (H\&E) stained liver resection specimens is reliable and replicable, it is invasive and the evaluation is difficult to perform before surgery (11). Therefore, it is necessary to develop an accurate noninvasive surrogate marker for this histopathological biomarker. Magnetic resonance imaging (MRI) is routinely performed in clinical practice, and has been shown to be more sensitive than other techniques, such as CT and PET/ CT, for the diagnosis of liver metastases $(12,13)$. MRI could identify the morphological characteristics of liver metastases, together with the surrounding liver parenchyma (14). However, the MRI performances of CRLM and the surrounding liver parenchyma vary greatly, and it is still unclear whether there are some differences in MRI performances between different HGPs.

The objective of this study was to identify the difference between the various HGPs of CRLM by MRI features. The underlying hypothesis was that HGPs differentiation through MRI would lead to a better therapeutic strategy selection and more accurate prognosis prediction, especially for patients with advanced cancers who cannot undergo surgery.

\section{Methods}

\section{Patients}

The study was conducted in accordance with the Declaration of Helsinki (as revised in 2013). This singleinstitution retrospective study was approved by the Ethical Committee of the First Hospital of China Medical University, and the informed consent was waived. Most patients with synchronous resectable liver metastases were treated with surgery after receiving neoadjuvant chemotherapy, and could not be included in the study. From December 2013 to September 2019, only 70 chemonaïve patients were confirmed as CRLM pathologically after hepatectomy. In the 70 patients, those without available specimens for histopathological assessment $(\mathrm{n}=8)$ and without MR examination within 4 weeks before surgery $(\mathrm{n}=9)$ were excluded. The clinical and pathological data were obtained from the medical records, including age, gender, location of primary cancer, time interval between hepatic MRI and hepatectomy, pathology of primary tumor, pathological TNM stage, and number of liver metastasis.

\section{Pathological analysis}

The HGPs were assessed on H\&E slices by two 
pathologists (WHL and XFW), both with more than 10 years of experience in gastrointestinal pathology. According to the international consensus guidelines (7), lesions were categorized as dHGP, rHGP, or pHGP when $>50 \%$ of the tumor-liver interface showed desmoplastic, replacement, or pushing appearance, respectively. Lesion was considered to be a mixed HGP if none of the three HGP was present in $>50 \%$ of the interface.

\section{MRI techniques}

All MR examinations were performed using a 3.0T MR scanner (Signa HDxt 3T, GE Healthcare) with an 8-channel surface coil for the body. Unenhanced MR examinations included a fast-spin-echo (FSE) T2-weighted sequence with fat saturation [repetition time (TR)/echo time (TE), 7,500/105], in-phase (TR/TE, 260/2.4) and out-phase (TR/TE, 260/3.2) T1-weighted gradient-echo (GE) sequences, with the section thickness of $6 \mathrm{~mm}, 1.2 \mathrm{~mm}$ intersection gap, matrix $224 \times 320$. Dynamic contrastenhanced MR examinations included one precontrast acquisition and five-postcontrast acquisitions by using a fatsuppressed T1-weighted three-dimensional fast-spoiled GE sequence (TR/TE, 3/1.3; flip angle, $15^{\circ} ; 5.0 \mathrm{~mm}$ section thickness; no gap; matrix $160 \times 276)$. Gadopentetate dimeglumine (Magnevist; Bayer Schering Pharma, Osaka, Japan) was injected into an antecubital vein by using an automated injector at a dose of $0.1 \mathrm{mmol}$ per kilogram of body weight and at a rate of $2 \mathrm{~mL} / \mathrm{sec}$ followed by a $20 \mathrm{~mL}$ saline flush. The early and late arterial phases were obtained at 15 and $35 \mathrm{~s}$ after administration of contrast medium, respectively. The portal phase images were obtained $35 \mathrm{~s}$ after the beginning of the arterial phase. The delayed phase images were obtained at about $240 \mathrm{~s}$ after contrast medium administration.

\section{MRI analysis}

Dynamic MRI were retrospectively reviewed by two abdominal imaging radiologists (SW and RMC), who were blinded to the histopathological information and the original imaging reports. The discrepancies between the two readers were resolved by discussion to reach consensus. We analyzed only one lesion for each patient. For multiple CRLMs, the MRI features were assessed on the lesion corresponding to the histopathological result if the specimens were from one lesion, while the largest one with pathological result was assessed if the specimens were from multiple lesions. A detailed assessment of the CRLM was documented, and the parameters were as follows: (I) the number of CRLM, (II) the longest tumor diameter (D) on precontrast T1-weighted images (T1WI), (III) the longest tumor diameter on arterial phase images $\left(\mathrm{D}_{\mathrm{a}}\right)$, (IV) tumor margin after enhancement: sharp (defined as a clear border between the tumor margin and the surrounding liver parenchyma) or unclear (no obvious border between the tumor margin and the surrounding liver parenchyma), $(\mathrm{V})$ the rim enhancement of tumor (appearing as a circumferential hyperintensity that is higher than central parts of the tumor and the surrounding liver parenchyma), and (VI) perilesional parenchymal enhancement (defined as wedge-shaped subsegmental or circumferential high signal intensity adjacent to or around the tumors distinguished from the background hepatic parenchyma on dynamic enhanced images). The diameter difference $(\Delta \mathrm{D})$ between the precontrast and postcontrast images was determined as: $\Delta \mathrm{D}=\mathrm{D}_{\mathrm{a}}-\mathrm{D}$. When a rim enhancement was present on postcontrast images, the width of the rim was measured, and the washout of the rim enhancement was recorded. Washout was defined as a decrease in enhancement, with isointensity or hypointensity during the portal and delayed phase. The measurement of tumor diameter and rim width was performed twice by a single reviewer (RMC), and the average data were recorded.

\section{Statistical analysis}

As rHGP is a strong independent prognostic marker in chemo-naïve patients according to previous reports (6-10), all the HGPs were classified into two groups in this research, namely rHGP and non-rHGP (including dHGP, pHGP and mixed HGP).

Continuous values are expressed as mean \pm standard deviation. The categorical features were compared by Chisquare test, while Student's $t$-test was used to compare the continuous values. The Spearman or Pearson correlation analysis with two-tailed testing of significance was performed to determine the correlation between the MRI features. A receiver operating characteristic (ROC) curve was plotted to evaluate the diagnostic ability. Statistical analysis was performed using $\mathrm{R}$ package (version 3.3.3, https://www.r-project.org/, R Foundation for Statistical Computing, Vienna, Austria). For all tests, $\mathrm{P}$ value $<0.05$ was considered statistically significant. 

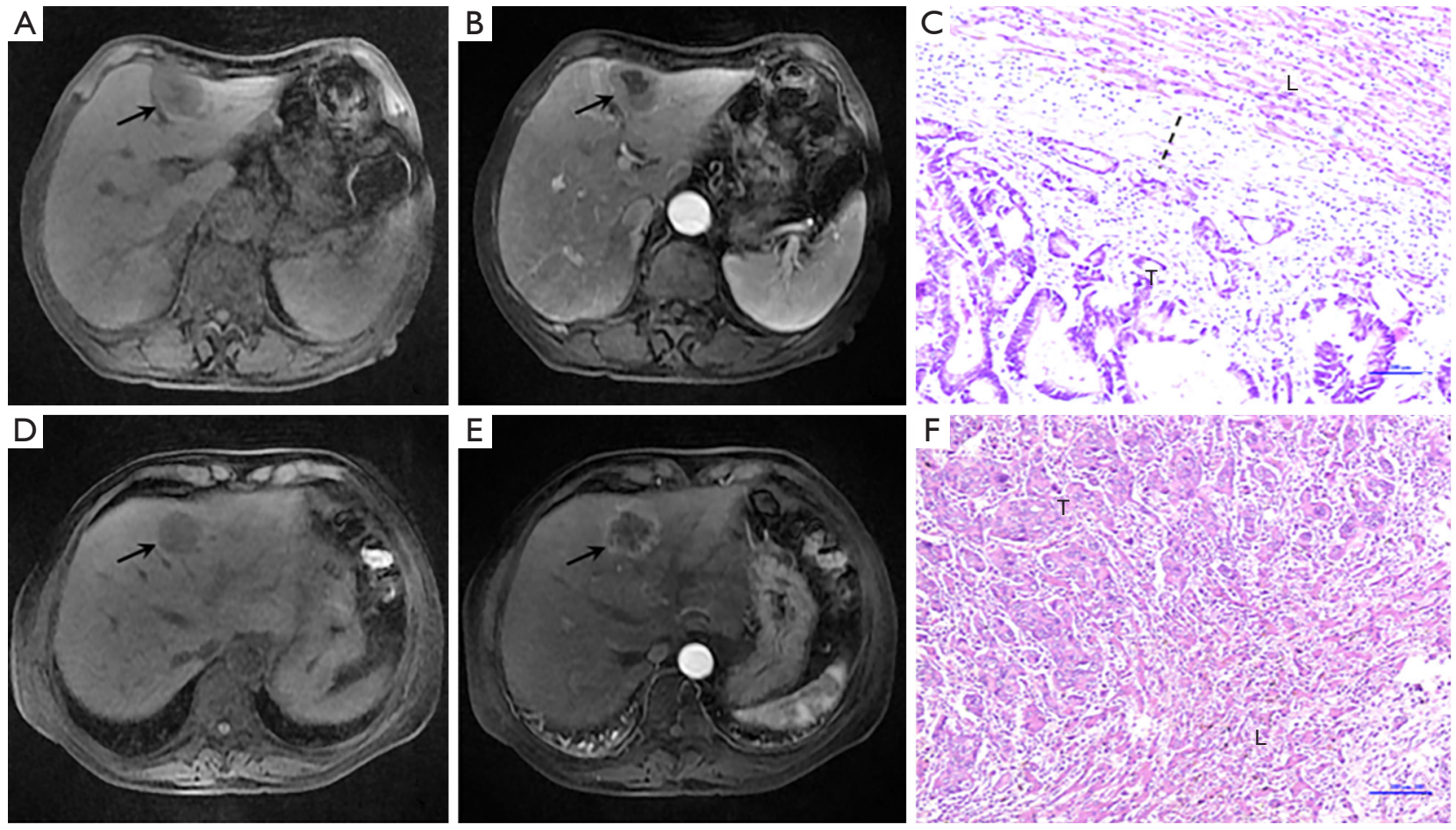

Figure 1 Images in an 86-year-old woman with CRLM of dHGP in the lateral segment of the left hepatic lobe (A,B,C). (A) Image of the precontrast T1WI shows a hypointense mass (arrow). (B) Arterial phase image demonstrates the mass has no rim enhancement at the margin (arrow). (C) Microscopic view (HE, ×200) of the histologic specimen shows the nodule of metastatic, moderately differentiated adenocarcinoma $(\mathrm{T})$ is separated from the hepatic parenchyma $(\mathrm{L})$ by fibrous stroma (dashed line). Images in a 51-year-old woman with CRLM of rHGP in the medial segment of the left hepatic lobe (D-F). (D) Image of the precontrast T1WI shows a hypointense mass (arrow). (E) Arterial phase image demonstrates the mass has an intense rim enhancement at the margin (arrow). (F) Microscopic view (H\&E stain; original magnification, $\times 200$ ) of the histologic specimen shows the nodule of metastatic, poorly differentiated adenocarcinoma (T) is in continuity with the hepatic parenchyma (L), without fibrous stroma. CRLM, colorectal liver metastasis; dHGP, desmoplastic histopathological growth pattern; T1WI, T1-weighted images; rHGP, replacement histopathological growth pattern; H\&E, hematoxylinand-eosin.

\section{Results}

Fifty-three chemo-naïve patients were enrolled in this study (mean age, $60.11 \pm 9.85$ years; age range, $38-86$ years), in which 31 were male, and 22 were female. The mean time from MRI to surgery was $9.01 \pm 5.38$ (range, 1-28) days. Left-sided colorectal cancer was noted in 40 patients, and right-sided colorectal cancer was noted in 13 patients. According to the international consensus guidelines, the rHGP was predominant in $12(22.64 \%)$ of the included CRLMs, the dHGP in 25 (47.17\%) tumors and the pHGP in $16(30.19 \%)$ tumors. No mixed HGP was identified. MR images and pathological images of typical cases were shown in Figure 1. No significant differences were detected in the gender, age, interval between MRI and surgery, location of primary tumor, pathology of primary tumor, $\mathrm{T}$ stage, and $\mathrm{N}$ stage between patients in the rHGP and non-rHGP groups, as shown in Table 1.

The diameter of the tumor ranged from 0.50 to 11.00 (mean, $4.28 \pm 0.25$ ) $\mathrm{cm}$. Thirty-one CRLMs showed rim enhancement in the arterial phase images, 12 were rHGP while 19 were non-rHGP. All the CRLMs with rHGP showed rim enhancement, while only $46.34 \%$ lesions with non-rHGP showed rim enhancement. The difference was significant $(\mathrm{P}<0.001)$. Besides, the $\Delta \mathrm{D}$ of rHGP was significantly larger than that of the non-rHGP $(\mathrm{P}=0.001)$. The tumor diameter on both precontrast T1WI and arterial phase images, number of CRLM, tumor margin after enhancement, and perilesional parenchymal enhancement were not different between the two groups $(\mathrm{P}>0.117$, Table 2). 
Table 1 Clinicopathological characteristics of patients in the rHGP and non-rHGP group

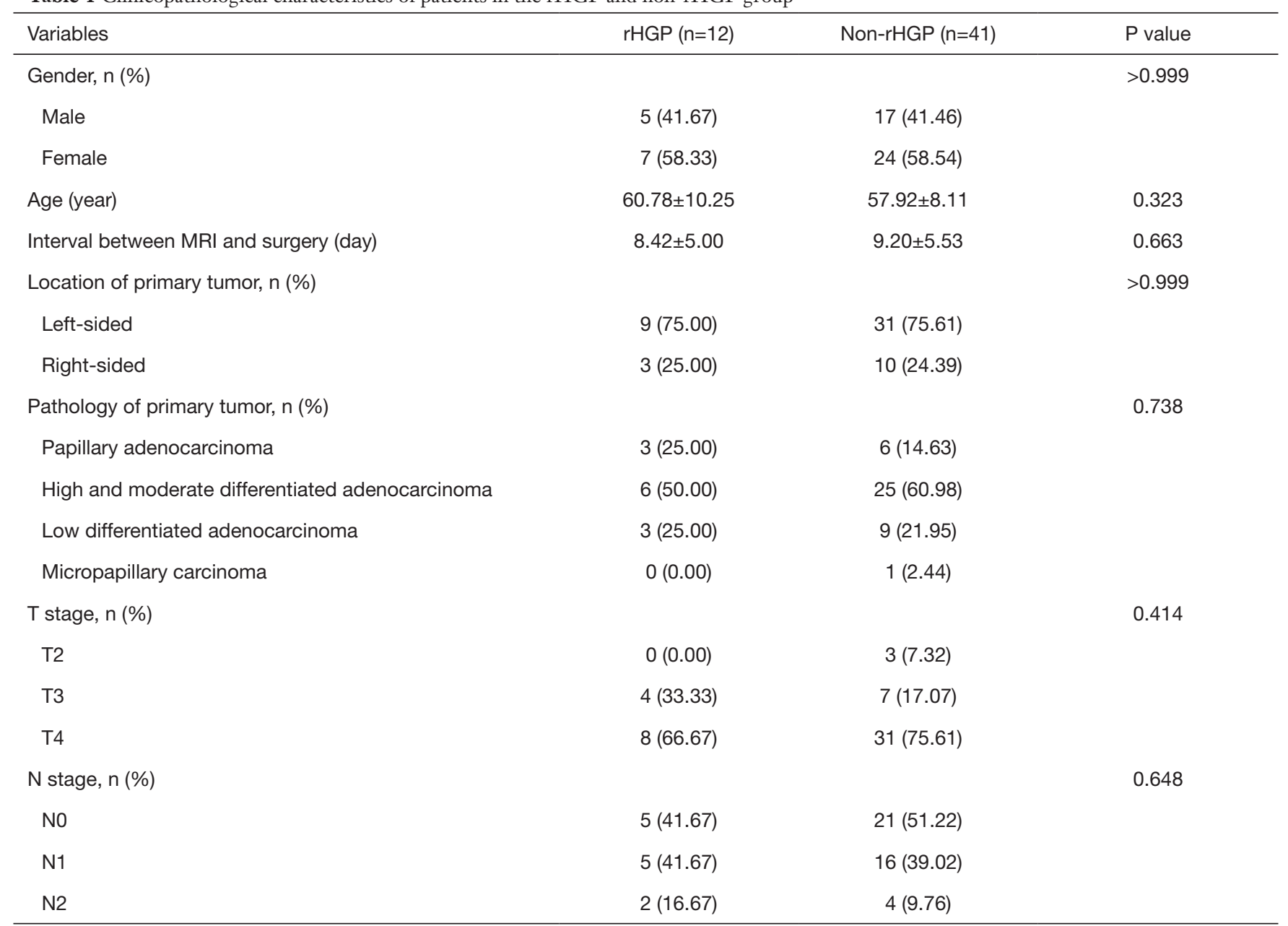

Non-rHGP: including the desmoplastic histopathological growth pattern and the pushing histopathological growth pattern. rHGP, replacement histopathological growth pattern; MRI, magnetic resonance imaging.

For the lesions with rim enhancement, the width of rim on arterial phase MRI ranged from 0.20 to 0.91 (mean, $0.49 \pm 0.22) \mathrm{cm}$ (Table 3). In these cases, the correlation coefficient of the rim width and the $\Delta \mathrm{D}$ was $0.584(\mathrm{P}<0.001)$. However, the width of rim was not correlated with the tumor diameter $(\mathrm{P}=0.262)$. The $\triangle \mathrm{D}$ of $\mathrm{rHGP}$ was greater than that of non-rHGP, though the difference was not significant $(\mathrm{P}=0.060)$. On delayed phase images, the rim enhancement disappeared in 7/12 rHGP and 17/19 in nonrHGP, suggesting that the CRLMs of non-rHGP were prone to be washed out in the delayed phase $(\mathrm{P}=0.043)$. The width of rim and the tumor margin after enhancement had no difference between the two groups $(\mathrm{P}>0.740)$.

Using multivariate analysis, the ROC curve was made for differentiating rHGP from non-rHGP by using a combination of rim enhancement and $\Delta \mathrm{D}$ which were significantly different between the two groups, as shown in Figure 2. The area under the curve (AUC) was 0.828 (95\% CI: 0.708-0.949).

\section{Discussion}

Approximately $50-60 \%$ of patients diagnosed with colorectal cancer develop metastases, and $80-90 \%$ of these patients have unresectable liver metastases (3). For the advanced or metastatic disease that is not amenable to resection, a systemic therapy is recommended. It has been shown that bevacizumab combined with chemotherapy (bev-chemo) can extend progression-free and/or overall survival (3). However, the anti-angiogenic 
Table 2 MRI features of the analyzed liver metastases

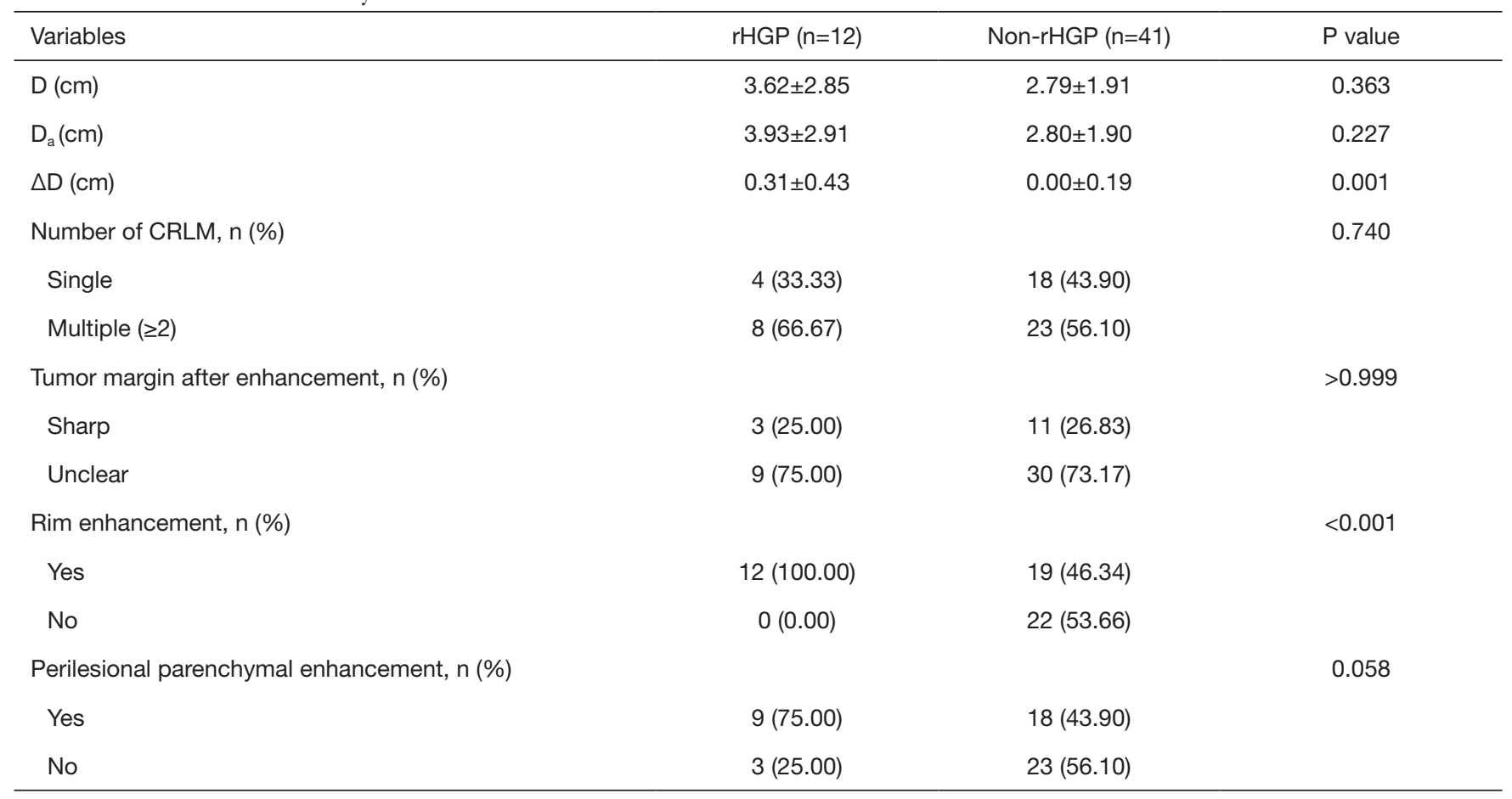

Non-rHGP: including the desmoplastic histopathological growth pattern and the pushing histopathological growth pattern; D: the longest tumor diameter on precontrast T1Wl; $\mathrm{D}_{\mathrm{a}}$ : the longest tumor diameter on arterial phase images; $\triangle \mathrm{D}$ : the tumor diameter difference between the precontrast and arterial phase images. MRI, magnetic resonance imaging; rHGP, replacement histopathological growth pattern; CRLM, colorectal liver metastasis; T1WI, T1-weighted images.

Table 3 Variables correlated with rim enhancement

\begin{tabular}{lccc}
\hline Variables & rHGP $(\mathrm{n}=12)$ & Non-rHGP $(\mathrm{n}=19)$ & $\mathrm{P}$ value \\
\hline $\mathrm{D}(\mathrm{cm})$ & $3.62 \pm 2.85$ & $2.95 \pm 2.152$ & 0.467 \\
$\mathrm{D}_{\mathrm{a}}(\mathrm{cm})$ & $3.93 \pm 2.91$ & $3.03 \pm 2.13$ & 0.332 \\
$\Delta \mathrm{D}(\mathrm{cm})$ & $0.31 \pm 0.43$ & $0.07 \pm 0.22$ & 0.060 \\
Rim width $(\mathrm{cm})$ & $0.49 \pm 0.22$ & $0.47 \pm 0.17$ & \\
Tumor margin after enhancement, $\mathrm{n}(\%)$ & & $5(26.31)$ \\
Sharp & $3(25.00)$ & $14(73.68)$ \\
$\quad$ Unclear & $9(75.00)$ & & 0.935 \\
Washout of rim enhancement, $\mathrm{n}(\%)$ & & $17(89.47)$ \\
Yes & $7(58.33)$ & $2(10.53)$ \\
No & $5(41.67)$ & 0.043 \\
\hline
\end{tabular}

Non-rHGP: including the desmoplastic histopathological growth pattern and the pushing histopathological growth pattern; D: the longest tumor diameter on precontrast $\mathrm{T} 1 \mathrm{Wl} ; \mathrm{D}_{\mathrm{a}}$ : the longest tumor diameter on arterial phase images; $\triangle \mathrm{D}$ : the tumor diameter difference between the precontrast and arterial phase images. rHGP, replacement histopathological growth pattern; T1WI, T1-weighted images. 


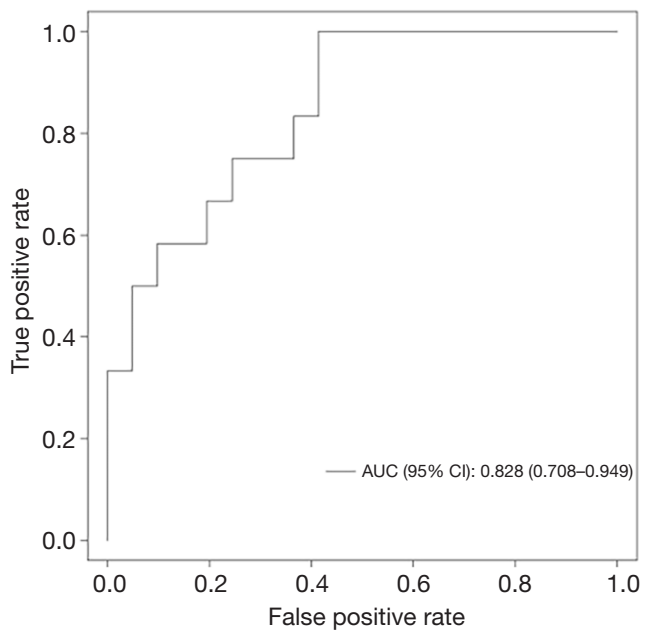

Figure 2 ROC curve for the differentiation of rHGP and nonrHGP by using rim enhancement and $\Delta \mathrm{D}$. ROC, receiver operating characteristic; rHGP, replacement histopathological growth pattern; $\Delta \mathrm{D}$, diameter difference.

agents (including bevacizumab) were designed to target sprouting angiogenesis, other than target the process of vessel co-option (5). Thus, the rHGP with vessel co-option is a potential resistance to anti-angiogenic therapy. An accurate prediction of HGP of CRLM could help to avoid unnecessary treatment. The current study demonstrates that MRI performance is different between rHGP and nonrHGP CRLMs. The rim enhancement is more common in rHGP, and the $\triangle \mathrm{D}$ between arterial phase image and precontrast image is larger in rHGP than in non-rHGP. This study shows that it is possible to stratify CRLMs of different HGP by using MRI features.

Liver metastasis is not difficult to diagnose on MRI combined with the history of colorectal cancer. The typical MRI feature of CRLM was described as rim enhancement on arterial phase images, with incomplete central progression on delayed phase images $(14,15)$. Researchers have been taking a great interest in the diagnostic value of rim enhancement of hepatic metastases on MRI. A study carried by $\mathrm{Yu}$ et al. (16), who measured the change of tumor size after enhancement, proposed that the main component of the rim enhancement is mainly at the extralesional area rather than tumoral area. In our study, we observed that the tumor diameter of rHGP was larger on arterial phase images than on precontrast images; it was in consistent with the previous research. However, the diameter of nonrHGP CRLMs with rim enhancement did not change much after enhancement. Although the $\Delta \mathrm{D}$ were different, the widths of rim enhancement between rHGP and non-rHGP CRLMs were almost the same. This may suggest that the rim enhancement of rHGP is mainly outside the tumor, while the rim enhancement of non-rHGP is mainly inside the tumor.

The biological mechanism of the association between MRI performance and HGP remains unclear. Early research revealed that the rim enhancement is related with histopathologic hepatic parenchymal changes, which include peritumoral desmoplastic reaction, inflammatory cell infiltration, and vascular proliferation (17). The recent studies further provided new histological evidences for the different tumor-liver interface between different HGPs $(5,7,18)$. In the CRLMs with dHGP and pHGP, they utilize angiogenesis to obtain vascular supply. The newly formed arterioles are tortuous, disrupted and leaking, thus the perfusion efficiency is low. However, in the rHGP CRLMs, the tumor cells infiltrate the liver parenchyma, and the intratumoral vessels co-opt with the sinusoidal blood supply of the liver parenchyma, without inducing sprouting angiogenesis. Compared with dHGP metastases, the microvessel density in CRLMs with rHGP is significantly higher at the tumor-liver interface (18). Moreover, the coopted capillary bed from normal liver is highly efficient and liver metastases with rHGP display higher perfusion as opposed to the CRLMs with non-rHGP (19). We observed that the CRLMs of rHGP had more rim enhancement compared with the non-rHGP type. This may indicate that the rim enhancement on MRI is more related with vascularization and perfusion of the tumor-liver interface. As for the location, the rim enhancement of rHGP may be related with the surrounding sinusoidal blood supply, therefore, it may include some adjacent liver parenchyma; while the blood supply of non-rHGP is mainly new formed blood vessels, thus the rim enhancement may be mainly inward the lesion.

In recent years, radiomics method is very popular in tumor research. Cheng et al. (20) retrospectively analyzed 68 dHGP and 58 rHGP CRLMs, and demonstrated that radiomics model derived from the CT images could be used to differentiate HGPs of CRLM. In their study, they found that the rim enhancement of dHGP is more common than that of rHGP, which were just in contrary with our results. They divided their sample into two sets: training set and validation set. There was a significant difference in rim enhancement in the training set, but no difference in the validation set. The grouping method may exaggerate 
the difference in one set. Han et al. (21) took an MRIbased radiomics study on 182 CRLMs, which include 59 dHGP and 123 rHGP CRLM lesions, and also proved that MRI-based radiomics method has the potential to determine the HGPs of CRLM. They reported that the rHGP $(25 \%)$ tends to exhibit a lobular margin compared with dHGP (17.1\%), and they did not find the difference of rim enhancement between dHGP and rHGP. The different result may also be related to the fact that they divided the sample into three subsets: training set, internal validation set, and external validation set. Besides, we only analyzed one lesion corresponding with the pathological result in multiple CRLMs, while Cheng et al. (20) and Han et al. (21) both included all the lesions in multiple CRLMs. There are only few studies investigated the pathological heterogeneity in multiple lesions and the results are inconsistent. Some studies $(4,22)$ showed similar pathological features in multiple lesions, while some studies $(18,23)$ reported that multiple CRLMs may show more than one HGP. It is still unclear whether the imaging features of multiple lesions are the same. The different inclusion criteria may also be one of the reasons why our results are different from previous studies.

There were several limitations to this study. First, this was a retrospective study performed at a single institution, and the sample size was relatively small. Second, the study did not include diffusion-weighted imaging (DWI) and hepatobiliary phase images, although these two sequences have been reported to be very sensitive for detecting liver metastases, especially for lesions smaller than $10 \mathrm{~mm}$ (24). Because some patients in the study did not receive DWI or hepatobiliary specific examination, we only analyzed the routine MR images of precontrast and postcontrast T1WI. The value of DWI and hepatobiliary phase images for differentiating dHGP and non-dHGP still needs further study.

In summary, CRLMs of rHGP and non-rHGP perform different on MRI. Compared with non-rHGP, more CRLMs of rHGP showed a rim enhancement, and rHGP CRLMs were enlarged more than non-rHGP ones on postcontrast images. By using the MRI feature to identify HGPs, it may be possible to stratify the patients prior to treatment so that they can benefit from personalized therapeutic plan.

\section{Acknowledgments}

Funding: None.

\section{Footnote}

Conflicts of Interest: All authors have completed the ICMJE uniform disclosure form (available at https://dx.doi. org/10.21037/qims-21-143). The authors have no conflicts of interest to declare.

Ethical Statement: The authors are accountable for all aspects of the work in ensuring that questions related to the accuracy or integrity of any part of the work are appropriately investigated and resolved. The study was conducted in accordance with the Declaration of Helsinki (as revised in 2013). The study was approved by the Institutional Research Ethics Board of the First Affiliated Hospital of China Medical University, and individual consent for this retrospective analysis was waived.

Open Access Statement: This is an Open Access article distributed in accordance with the Creative Commons Attribution-NonCommercial-NoDerivs 4.0 International License (CC BY-NC-ND 4.0), which permits the noncommercial replication and distribution of the article with the strict proviso that no changes or edits are made and the original work is properly cited (including links to both the formal publication through the relevant DOI and the license). See: https://creativecommons.org/licenses/by-nc-nd/4.0/.

\section{References}

1. Benson AB, Venook AP, Al-Hawary MM, Cederquist L, Chen YJ, Ciombor KK, et al. Rectal cancer, version 2.2018, NCCN clinical practice guidelines in oncology. J Natl Compr Canc Netw 2018;16:874-901.

2. Hayashi M, Inoue Y, Komeda K, Shimizu T, Asakuma M, Hirokawa F, Miyamoto Y, Okuda J, Takeshita A, Shibayama Y, Tanigawa N. Clinicopathological analysis of recurrence patterns and prognostic factors for survival after hepatectomy for colorectal liver metastasis. BMC Surg 2010;10:27.

3. Benson AB, Venook AP, Al-Hawary MM, Arain MA, Chen YJ, Ciombor KK, et al. Colon cancer, version 2.2021, NCCN clinical practice guidelines in oncology. J Natl Compr Canc Netw 2021;19:329-59.

4. Terayama N, Terada T, Nakanuma Y. Histologic growth patterns of metastatic carcinomas of the liver. Jpn J Clin Oncol 1996;26:24-9.

5. Frentzas S, Simoneau E, Bridgeman VL, Vermeulen PB, Foo S, Kostaras E, et al. Vessel co-option mediates 
resistance to anti-angiogenic therapy in liver metastases. Nat Med 2016;22:1294-302.

6. Pinheiro RS, Herman P, Lupinacci RM, Lai Q, Mello ES, Coelho FF, Perini MV, Pugliese V, Andraus W, Cecconello I, D'Albuquerque LC. Tumor growth pattern as predictor of colorectal liver metastasis recurrence. Am J Surg 2014;207:493-8.

7. van Dam PJ, van der Stok EP, Teuwen LA, Van den Eynden GG, Illemann M, Frentzas S, et al. International consensus guidelines for scoring the histopathological growth patterns of liver metastasis. Br J Cancer 2017;117:1427-41.

8. Galjart B, Nierop PMH, van der Stok EP, van den Braak R, Hoppener DJ, Daelemans S, Dirix LY, Verhoef C, Vermeulen PB, Grunhagen DJ. Angiogenic desmoplastic histopathological growth pattern as a prognostic marker of good outcome in patients with colorectal liver metastases. Angiogenesis 2019;22:355-68.

9. Siriwardana PN, Luong TV, Watkins J, Turley H, Ghazaley M, Gatter K, Harris AL, Hochhauser D, Davidson BR. Biological and prognostic significance of the morphological types and vascular patterns in colorectal liver metastases (CRLM): looking beyond the tumor margin. Medicine (Baltimore) 2016;95:e2924.

10. Eefsen RL, Vermeulen PB, Christensen IJ, Laerum OD, Mogensen MB, Rolff HC, Van den Eynden GG, HøyerHansen G, Osterlind K, Vainer B, Illemann M. Growth pattern of colorectal liver metastasis as a marker of recurrence risk. Clin Exp Metastasis 2015;32:369-81.

11. Höppener DJ, Nierop PMH, Herpel E, Rahbari NN, Doukas M, Vermeulen PB, Grünhagen DJ, Verhoef C. Histopathological growth patterns of colorectal liver metastasis exhibit little heterogeneity and can be determined with a high diagnostic accuracy. Clin Exp Metastasis 2019;36:311-9.

12. Sivesgaard K, Larsen LP, Sorensen M, Kramer S, Schlander S, Amanavicius N, Bharadwaz A, Tonner Nielsen D, Viborg Mortensen F, Morre Pedersen E. Diagnostic accuracy of CE-CT, MRI and FDG PET/CT for detecting colorectal cancer liver metastases in patients considered eligible for hepatic resection and/or local ablation. Eur Radiol 2018;28:4735-47.

13. Niekel MC, Bipat S, Stoker J. Diagnostic imaging of colorectal liver metastases with CT, MR imaging, FDG PET, and/or FDG PET/CT: a meta-analysis of prospective studies including patients who have not previously undergone treatment. Radiology 2010;257:674-84.
14. Danet IM, Semelka RC, Leonardou P, Braga L, Vaidean G, Woosley JT, Kanematsu M. Spectrum of MRI appearances of untreated metastases of the liver. AJR Am J Roentgenol 2003;181:809-17.

15. Namasivayam S, Martin DR, Saini S. Imaging of liver metastases: MRI. Cancer Imaging 2007;7:2-9.

16. Yu JS, Rofsky NM. Hepatic metastases: perilesional enhancement on dynamic MRI. AJR Am J Roentgenol 2006;186:1051-8.

17. Semelka RC, Hussain SM, Marcos HB, Woosley JT. Perilesional enhancement of hepatic metastases correlation between MR imaging and histopathologic findings-initial observations. Radiology 2000;2 15:89-94.

18. Lazaris A, Amri A, Petrillo SK, Zoroquiain P, Ibrahim N, Salman A, Gao Z-H, Vermeulen PB, Metrakos P. Vascularization of colorectal carcinoma liver metastasis: insight into stratification of patients for anti-angiogenic therapies. J Pathol Clin Res 2018;4:184-192.

19. Stessels F, Van den Eynden G, Van der Auwera I, Salgado R, Van den Heuvel E, Harris AL, Jackson DG, Colpaert CG, van Marck EA, Dirix LY, Vermeulen PB. Breast adenocarcinoma liver metastases, in contrast to colorectal cancer liver metastases, display a non-angiogenic growth pattern that preserves the stroma and lacks hypoxia. Br J Cancer 2004;90:1429-36.

20. Cheng J, Wei J, Tong T, Sheng W, Zhang Y, Han Y, Gu D, Hong N, Ye Y, Tian J, Wang Y. Prediction of histopathologic growth patterns of colorectal liver metastases with a noninvasive imaging method. Ann Surg Oncol 2019;26:4587-98.

21. Han Y, Chai F, Wei J, Yue Y, Cheng J, Gu D, Zhang Y, Tong T, Sheng W, Hong N, Ye Y, Wang Y, Tian J. Identification of predominant histopathological growth patterns of colorectal liver metastasis by multi-habitat and multi-sequence based radiomics analysis. Front Oncol 2020;10:1363.

22. Okano K, Yamamoto J, Kosuge T, Yamamoto S, Sakamoto M, Nakanishi Y, Hirohashi S. Fibrous pseudocapsule of metastatic liver tumors from colorectal carcinoma. Clinicopathologic study of 152 first resection cases. Cancer 2000;89:267-75.

23. Eefsen RL, Van den Eynden GG, Høyer-Hansen G, Brodt P, Laerum OD, Vermeulen PB, Christensen IJ, Wettergren A, Federspiel B, Willemoe GL, Vainer B, Osterlind K, Illemann M. Histopathological growth pattern, proteolysis and angiogenesis in chemonaive patients resected for multiple colorectal liver metastases. J 
Oncol 2012;2012:907971.

24. Kim HJ, Lee SS, Byun JH, Kim JC, Yu CS, Park SH, Kim AY,Ha HK. Incremental value of liver MR imaging in patients with potentially curable colorectal hepatic metastasis detected at CT. Radiology 2015;274:712-22.

Cite this article as: $\mathrm{Li} \mathrm{WH}$, Wang $\mathrm{S}$, Liu $\mathrm{Y}$, Wang $\mathrm{XF}$, Wang YF, Chai RM. Differentiation of histopathological growth patterns of colorectal liver metastases by MRI features. Quant Imaging Med Surg 2022;12(1):608-617. doi: 10.21037/ qims-21-143 